\title{
LA FORMACIÓN CIENTÍFICA Y EJERCICIO PROFESIONAL EN ESPAÑA DE VICENTE CERVANTES MENDO
}

\author{
José Pastor Villegas* \\ Departamento de Química Orgánica e Inorgánica. Universidad de Extremadura
}

\begin{abstract}
RESUMEN
Vicente Cervantes Mendo (Ledrada, Salamanca, 1758-México, 1829) fue un insigne científico hispanomexicano; hoy es patrimonio de España y México. Como una continuación de dos artículos recientes sobre su vida en España, este artículo trata de su formación científica, como farmacéutico y como botánico, y de su ejercicio profesional en Madrid. Dos informes de Casimiro Gómez Ortega de 1786, catedrático primero del Real Jardín Botánico de Madrid, en relación con la Real Expedición Botánica a Nueva España (1787-1803), han servido para establecer conclusiones que precisan los aspectos estudiados y corrigen errores.
\end{abstract}

PALABRAS CLAVE: Vicente Cervantes Mendo. Real Jardín Botánico de Madrid. Reales Hospitales de Madrid. Farmacia. Botánica.

\section{SCIENTIFIC TRAINING AND PROFESSIONAL PRACTICE IN VICENTE CERVANTES MENDO'S SPAIN}

\begin{abstract}
Vicente Cervantes Mendo (Ledrada, Salamanca, 1758-México, 1829) was a famous Spanish Mexican scientific; he is today heritage of Spain and Mexico. As a continuation of two recent articles on his life at Spain, the present study deals with his scientific formation at Madrid, as pharmacist and as botanist, as well as on his professional activity. Two documents of Casimiro Gómez Ortega, principal professor of the Real Jardín Botánico de Madrid, dated in 1786 and related with the Real Expedición Botánica a Nueva España (1787-1803), have served to establish conclusions which clarify the subjects studied and correct mistakes.
\end{abstract}

KEY WORDS: Vicente Cervantes Mendo. Real Jardín Botánico de Madrid. Reales Hospitales de Madrid. Pharmacy. Botany.

* Información de contacto: José Pastor Villegas. Departamento de Química Orgánica e Inorgánica. Facultad de Formación del Profesorado. Universidad de Extremadura, Avda. de la Universidad s/n, 10071 Cáceres. Teléfono: + 34 927257050. Fax: + 34 927257051. E-mail: jpastor@unex.es. 


\section{INTRODUCCIÓN}

En la I Reunión de Historia de la Ciencia y de la Técnica de los Países Ibéricos e Iberoamericanos se enfatizó que lengua y ciencia son nexos de estos países que permanecen con el tiempo ${ }^{1}$. Esto es una realidad al tratar de Vicente Cervantes Mendo (Ledrada, Salamanca, 1758-México, 1829)2 , conocido como Vicente Cervantes, quien figura en dos conocidos diccionarios como un insigne farmacéutico ${ }^{3}$ y botánico ${ }^{4}$, y a quien se le puede considerar también químico $^{5}$. Son numerosas las publicaciones, principalmente españolas y mexicanas, que tratan con mayor o menor extensión sobre este científico trascendental para España y México, patrimonio de ambas naciones.

He escrito que el mérito de este científico como integrante de la Real Expedición Botánica a Nueva España (1787-1803), realizada en los reinados de Carlos III y Carlos IV, fue conocido y reconocido pronto por el Real Colegio de Boticarios de Madrid y la Real Academia Médica Matritense. Estas instituciones le otorgaron las distinciones de Colegial Honorario y Socio Correspondiente, respectivamente. He escrito también que en México se celebró el Primer Coloquio Mexicano de Historia de la Ciencia en 1964, destacando Enrique Beltrán, y que durante la vida colonial fue evento de importancia científica el arribo de la mencionada expedición científica, que estableció el Real Jardín Botánico de México y dejó en Nueva España a Vicente Cervantes, primer catedrático de Botánica de México ${ }^{6}$.

1 Peset, J.L. (1985), Introducción. En PeSet, J.L. (ed.), La Ciencia Moderna y el Nuevo Mundo, Madrid, CSIC/Sociedad Latinoamericana de Historia de las Ciencias y de la Tecnología, pp. 5-10.

2 Pastor Villegas, J. (2007), Vicente Cervantes Mendo: lugar y fecha de nacimiento, bicentenario no conmemorado y próximo 250 aniversario, An. Real Acad. Nac. Farm., 73, pp. 747-762.

3 RoldÁn Guerrero, R. (1958-1976), Diccionario biográfico y bibliográfico de autores farmacéuticos españoles, Madrid, Gráficas Valera, vol. 1, pp. 659-661.

4 RoldÁn Guerrero (1958-1976), pp. 659-661; LóPEZ PiÑERO, J.M. (1983), Vicente Cervantes. En López Piñero, J.M., Glick, T.F., NAVArro Brotóns, V. y Portela Ramos, M., Diccionario histórico de la ciencia moderna en España, Barcelona, Ediciones Península, vol. 1, pp. 209-210.

5 Aceves Pastrana, P. (1993), Química, Botánica y Farmacia en la Nueva España a finales del siglo XVIII, México D.F., Universidad Autónoma Metropolitana; PASTOR VILLEGAS, J. y PASTOR VALLE, J.F. (2003), Páginas extremeñas sobre el caucho, Trujillo, La Coria, Fundación Xavier de Salas, pp. 94-97.

6 PAStor Villegas (2007), pp. 747-762. 
Cronológicamente, la vida de Vicente Cervantes Mendo transcurrió en España, virreinato de Nueva España y México independiente. Considerando que Vicente Cervantes Mendo nació con certeza en Ledrada (Salamanca, España) en 1758, que fue integrante de la mencionada expedición científica, y que después continuó en México hasta su muerte, se pueden distinguir tres etapas en su vida y obra: la primera etapa (1758-1787) es española, la segunda etapa (1787-1803), o etapa colonial, corresponde a la expedición científica novohispana, y la tercera etapa (1803-1829), o etapa hispano-mexicana, es posterior a esta expedición científica.

La primera etapa de su vida viene a coincidir con el reinado de Carlos III (1759-1788), reinado en el que hubo un renovado impulso de la ciencia española para superar el conocido "apagón científico» que se había producido en la anterior dinastía austriaca. En particular, la sanidad de la Ilustración Española se benefició de las reformas borbónicas, principalmente entre 1780 y 1800 , es decir, en los años finales del reinado de Carlos III y gran parte del reinado de Carlos IV ${ }^{7}$.

En 1864, José García Ramos, individuo de número del Real Colegio de Farmacéuticos de Madrid y Ayudante Profesor de la Real Botica, escribió por encargo de la mencionada corporación profesional una monografía sobre Vicente Cervantes, que leyó en la sesión de aniversario de 21 de agosto de $1864^{8}$. Al revisar esta publicación, he concluido que los datos del lugar y la fecha de nacimiento son erróneos ${ }^{9}$, y que otros datos biográficos aportados sobre su vida en España ${ }^{10}$, algunos imprecisos y otros erróneos, pasaron también a diccionarios y publicaciones diversas hasta el presente.

Este artículo, escrito en el año en que las comunidades científicas de España y México conmemorarán el 250 aniversario del nacimiento de Vicente Cervantes Mendo, y como una continuación de los dos artículos recientemente publicados, versa también sobre la etapa española mencionada. En concreto, el objetivo es revisar la información proporcionada en la monografía de José García Ramos sobre la formación inicial como boticario y botánico en el Madrid científico de los años finales del reinado de Carlos III, y de su breve

7 Calleja Folguera, M.C. (1988), La reforma sanitaria en la España Ilustrada, Madrid, Universidad Complutense de Madrid, pp. IX-XII.

8 García Ramos J. (1864), Elogio histórico del farmacéutico Don Vicente Cervantes, catedrático que fue de Botánica en la Universidad de México, Madrid, Imprenta R. Anoz.

9 PASTOR Villegas (2007), pp. 747-762.

10 Pastor Villegas (2008), Vicente Cervantes Mendo, insigne boticario, botánico y químico hispanomexicano: su vida en España, Revista de Estudios Extremeños, 64, pp. 413-424. 
actividad profesional antes de pasar al virreinato de Nueva España. Se revisa tal publicación porque ha sido fuente de publicaciones diversas que incluyen información sobre el científico estudiado, como son las publicaciones que se citan sobre la expedición botánica novohispana ${ }^{11}$.

Durante el trabajo de investigación, además del elogio histórico escrito por José García Ramos y otras publicaciones diversas, el autor ha consultado documentación que se conserva en los archivos madrileños de la Real Academia Nacional de Farmacia, Real Jardín Botánico, Museo Nacional de Ciencias Naturales y Archivo Regional de la Comunidad de Madrid. Todos los párrafos documentales reproducidos en las secciones que siguen se han escrito con ortografía actual.

\section{Informes de CASimiro Gómez ORTEGa conexos con la ReAl EXPedi- CIÓN BOTÁNICA A NuEVA ESPAÑA (1787-1803)}

El Madrid del reinado de Carlos III, sede de la corte y del imperio, no tuvo universidad; tuvo instituciones científicas de nuevo cuño, involucradas en la modernización borbónica del Estado, muchas de ellas sitas en torno al actual Paseo del Prado ${ }^{12}$. Una de tales instituciones fue el Real Jardín Botánico de Madrid, cuyo 250 aniversario se ha conmemorado en $2005^{13}$. Se ha señalado que el fin del Real Jardín Botánico de Madrid en su inauguración fue estudiar $\mathrm{y}$ cultivar las plantas medicinales ${ }^{14}$. Con el transcurso del tiempo, fue una

11 RicketT, H.W. (1947), The Royal Botanical Expedition to New Spain 1788-1820, Chronica botanica, 11, pp. 1-86; ARIAS DIVITO, J.C. (1968), Las expediciones cientificas españolas durante el siglo XVIII. Expedición botánica de Nueva España, Madrid, Ediciones Cultura Hispánica; ENGSTRAND, I.H.W. (1981), Spanish Scientists in the New World: The Eighteenth-Century Expeditions, Seattle, University of Washington Press; Maldonado Polo, J.L. (2000), La Expedición Botánica a Nueva España, 1786-1803: el Jardín Botánico y la Cátedra de Botánica, HMex, 1, pp. 5-56; LozoyA, X. (1984), Plantas y luces en México. La Real Expedición Botánica a Nueva España (1787-1803), Madrid, Ediciones del Serval. MALDONADO POLO, J.L. (2001), Las huellas de la razón. La expedición científica de Centroamérica, Madrid, CSIC.

12 BARATAs DíAz, L.A. (1996), El núcleo de instituciones científicas matritenses en el Paseo del Prado desde el siglo XVIII, Asclepio, 48, pp. 183-217; LAfuenTE, A. (1998), Guía del Madrid Cientifico, Madrid, Doce Calles/Comunidad de Madrid/ CSIC.

13 San Pío Aladrén, M.P. De (ed.) (2005), El Real Jardín Botánico de Madrid (17552005): Ciencia, Colección y Escuela, Madrid, Real Jardín Botánico, CSIC/Lunwerg/Caja Madrid Obra Social.

14 Peset, J.L. (1996), El Jardín Botánico de Madrid y sus relaciones con Francia, Asclepio, 48 , pp. 59-70. 
institución polivalente: huerto real, centro docente de sanitarios y de botánicos, abastecedor de plantas medicinales a la Real Botica, aliado del Real Tribunal del Protomedicato en la centralización administrativa sanitaria, rector de los estudios florísticos nacionales, modelo del resto de los jardines botáni$\cos$ y dirigente de las expediciones científicas ultramarinas ${ }^{15}$. En particular, tuvo importancia peculiar en la reforma farmacéutica y en las expediciones científicas a América, sobre todo durante los años 1771-1801 en que Casimiro Gómez Ortega fue catedrático primero ${ }^{16}$. Este científico cortesano ejecutó la política científica de la Corona, en lo referente a la Historia Natural en España y sus colonias ultramarinas ${ }^{17}$; incorporó la Botánica al proyecto ambicioso, costoso, utópico y, en cierta medida original, de inventariar y comercializar las riquezas coloniales ${ }^{18}$.

Como es ampliamente conocido, la Real Expedición Botánica a Nueva España (1787-1803) se realizó en los reinados de Carlos III y Carlos IV; fue una compleja e importante expedición científica española encabezada por el médico aragonés Martín Sessé y Lacasta, conexa en parte con la Primera Expedición Científica a América (1571-1577) que había realizado el médico y naturalista toledano Francisco Hernández en el reinado de Felipe II.

La génesis de la expedición botánica novohispana comienza con una carta del mencionado médico aragonés establecido en México, fechada en La Habana el 30 de enero de $1785^{19}$. En el Museo de Ciencias Naturales de Madrid hay dos informes de Casimiro Gómez Ortega ${ }^{20}$ cuando se estaba gestando esta expedición, uno de fecha 29 de marzo de 1786 y otro de fecha 21 de septiembre de 1786, que permiten precisar y corregir lo que se conoce sobre la formación científica y ejercicio profesional de Vicente Cervantes Mendo

15 Puerto Sarmiento, F.J. (1994), Química, Botánica y Farmacia en España a finales del siglo XVIII. En Aceves PastranA, P. (ed.), Química en Europa y América (siglos XVIII y XIX), México D.F., Universidad Autónoma Metropolitana, pp. 157-175.

16 Puerto SARmiento (1988), La ilusión quebrada. Botánica, sanidad y política cientifica en la España Ilustrada, Barcelona, SERVAL/CSIC, pp. 50-54; PUERTO SARMIENTO (1992), Ciencia de Cámara. Casimiro Gómez Ortega (1741-1818) el científico cortesano, Madrid, CSIC, pp. 50-78.

17 PUERTO SARMiento (1992), pp. 225-257.

18 GonZÁlez, A. (2005), El medicamento en el mundo moderno: las plantas sanadoras. En Esteva, J., Prieto, J. y Puerto, J., El Medicamento y la Vida Humana, Madrid, Ars Médica, pp. 42-52.

19 ARIAS DiVITO (1968), p. 53.

20 Calatayud Arinero, M.A. (1984), Catálogo de las expediciones y viajes científicos españoles, a América y Filipinas (siglos XVIII y XIX), Madrid, CSIC. 
en el Madrid de los años finales del reinado de Carlos III. La segunda fecha ha sido mencionada por Maldonado Polo al tratar del científico José Longinos Martínez Garrido ${ }^{21}$, sin hacer referencia alguna a Vicente Cervantes Mendo.

Con fecha 29 de marzo de 1786, Casimiro Gómez Ortega informó y dejó entrever en su informe la futura propuesta del joven Vicente Cervantes como integrante de la expedición científica que se comenzaba a organizar:

Que dicho joven con noticia de su nombramiento podrá acabar de perfeccionarse en toda la primavera y verano próximo, y llegar a México con ejemplares de la obra del Doctor Hernández, que para entonces estará ya impreso.

Concretamente, Casimiro Gómez Ortega propuso a Vicente Cervantes para integrar la expedición botánica novohispana el 21 de septiembre de 1786. En esta fecha proporciona también una información breve sobre su formación inicial en dos párrafos que se trascriben a continuación, con precisiones entre corchetes:

Propone Don Casimiro Ortega [Casimiro Gómez Ortega] que para entregarse en el Jardín Botánico [Real Jardín Botánico de México] y regentar la Cátedra de esta facultad cuando el Dr. Sesé [Sessé] salga a viajar, según expuso en su anterior informe, es muy a propósito Don Vicente Cervantes, que además de hallarse suficientemente instruido en los conocimientos para el efecto, ha estudiado Medicina, aunque no está graduado en ella, es buen Filósofo, buen químico, y farmacéutico; en cuya facultad no sólo está aprobado, sino que en concurso público en el Hospital General de Madrid, ha merecido la preferencia sobre todos sus competidores; posee la lengua francesa, y es hombre de carrera, de capacidad, de instrucción, y de lucimiento como lo manifestará en las inmediatas oposiciones de Botánica que se están disponiendo para el regreso de la Corte.

Y que dicho Cervantes luego que llegue a México podrá abrir el curso de lecciones enseñando los fundamentos teóricos mientras se dispone el Jardín para continuar la explicación de la Práctica, para lo cual se le nombrará con la condición de que no ha de restituirse a España hasta que se haya concluido la expedición del Dr. Sesé [Sessé], y haya formado discípulos capaces de sucederle.

Como se puede observar en el primero de estos párrafos, Casimiro Gómez Ortega afirma que Vicente Cervantes había estudiado Medicina, que era un

21 Maldonado Polo, J.L (1997), La aventura ultramarina de un naturalista calagurritano, Kalakoricos, 2, pp. 135-152. En la página 138 de este artículo figura la nota «3. Informe de Casimiro Gómez Ortega, Madrid, 21 de septiembre de 1786, Museo Nacional de Ciencias Naturales (MNCN), Flora Mexicana, leg.2, carp.3». 
buen filósofo, buen químico, buen farmacéutico y que antes de finalizar 1786 realizaría los ejercicios públicos botánicos. En las tres secciones que siguen se trata de su formación como boticario y botánico, y de su ejercicio profesional en Madrid.

\section{Boticario por el Real Tribunal del Protomedicato en El Madrid DE LOS AÑOS FINALES DEL REINADO DE CARLOS III}

Es sabido que a Madrid, centro del poder político desde el siglo XVI, llegaron gentes de muchos lugares. El ledradense Vicente Cervantes Mendo se formó y obtuvo el título de boticario en el Madrid de los años finales del reinado de Carlos III. José García Ramos ${ }^{22}$, considerándole nacido en Zafra, escribió varios párrafos sobre su formación y examen de boticario. Tales párrafos, que contienen información imprecisa, se resumen a continuación para precisarlos seguidamente.

Su primera diligencia luego que llegó a Madrid, fue buscar una Botica donde le admitiesen como dependiente y discípulo, y en la cual, a la vez que pudiera aprender la parte empírica de la Profesión, dispusiese del tiempo necesario para asistir a las lecciones de Botánica, por cuya ciencia tenía una especial predilección. No se le mostró la fortuna, en este caso, completamente adversa, pues su petición más esencial la vio pronto satisfecha, encontrando colocación en una Botica; pero [...] Cervantes no pudo asistir, como eran sus deseos, a las lecciones de Botánica [...]. Muy cerca de su Botica, se hospedaba un joven, amigo suyo, el cual asistía diariamente a las lecciones botánicas de D. Casimiro Gómez Ortega, catedrático y director entonces del Jardín botánico, y Cervantes pudo lograr de su amistad que pasase todos los días por su casa a manifestarle particularidades que la lección hubiese ofrecido para tenerlas él presentes y poder seguir particularmente la misma marcha y encontrarse a la misma altura que los demás compañeros. Pasó algún tiempo [...], hasta que un día, sintiéndose animado por las fuerzas que presta el convencimiento del propio saber, cuando este carece de arrogancia, se presentó al citado G. Ortega, que también era a la sazón presidente de este Colegio y alcalde examinador del Protomedicato, pidiendo se le admitiese a examen de farmacéutico a título de suficiencia y exponiéndole los motivos por los cuales no le había sido posible asistir a las lecciones para obtener las certificaciones correspondientes. Quedó suspenso Gómez Ortega [...] y se encargó de proteger su solicitud, no sin advertirle de antemano que, caso de conseguir lo que pedía, tuviese entendido que los ejercicios de

22 GARCÍA RAMOS, J. (1864), pp. 6-8. 
examen habrían de ser tan excesivamente rígidos, como corresponde a la magnitud de la gracia dispensada.

Así sucedió en efecto [...]. Sus respuestas fueron tan exactas y precisas, los argumentos tan victoriosamente refutados, las teorías tan bellamente desenvueltas, sus ejercicios, en fin, tan brillantes, que el tribunal, unánimemente admirado, le aclamó por ser muy digno de pertenecer a la clase farmacéutica, ordenando en su virtud que se le expidiese, como se verificó, el título correspondiente.

Para precisar estos párrafos conviene tener en cuenta cómo era la formación y examen de boticarios durante la Ilustración Española. En particular, en los años finales del reinado de Carlos III, en los cuales la profesión farmacéutica no era todavía profesión universitaria.

La enseñanza de la Farmacia en España fue problemática en el siglo $\mathrm{XVIII}^{23}$, y hasta principios del siglo XIX no existieron enseñanzas oficiales de esta ciencia ${ }^{24}$, es decir, cuando experimenta su revolución, recogiendo los avances de sus ciencias afines: Botánica y Química ${ }^{25}$. La instrucción del aprendiz de boticario fue competencia del boticario hasta muy avanzado el siglo XVIII ${ }^{26}$; por consiguiente, la formación de tal aprendiz dependía del maestro boticario. Innovación importante en ese siglo fue la creación de los Colegios de Boticarios, que fomentaron el desarrollo científico de la profesión. El Real Colegio de Boticarios de Madrid, fundado en 1732 e instituido oficialmente en 1737 (corporación antecedente remoto de la Real Academia de Farmacia del Instituto de España), tuvo carácter científico-profesional, con interés en el progreso de la Farmacia, Química, Botánica e Historia Natural ${ }^{27}$; publicó la Farmacopoeia Matritensis en 1739 con validez en toda España, así como la segunda edición en $1762^{28}$.

Los ilustrados españoles, impotentes ante la reforma universitaria, pero tenaces en su propósito de modernizar la sociedad, propiciaron las ciencias

23 Folch, G. (1982), Problemática de las enseñanzas de farmacia en la España del siglo XVIII. Intervención de los Colegios, An. Real Acad. Farm., 48, pp. 285-302.

24 Folch (1977), El Real Colegio de Farmacia de San Fernando, Madrid, Instituto de España, Real Academia de Farmacia.

25 Calleja Folguera, M.C. (1992), La Farmacia en la Ilustración. En Puerto SarMIENTO, F.J. (dir.), Historia de la Ciencia y de la Técnica, n. 31, Madrid, Akal, p. 7.

26 Folch ANDREU, R. (1941), La instrucción farmacéutica durante el siglo XVIII en los Hospitales Generales de Madrid, An. Real Acad. Farm., 1, pp. 27-57.

27 Estatutos del Real Colegio de Profesores Boticarios de Madrid, aprobados y confirmados por su Magestad, que Dios guarde (1737), Madrid, Imprenta Real, Estatuto 1, p. 4.

28 FrancÉs, M.C. y Aliaga, M.J. (2001), Intervención del Real Colegio de Farmacéuticos de Madrid en la edición de la Farmacopea Matritense, An. Real Acad. Farm., 67, pp. 1-16. 
útiles al margen de la universidad ${ }^{29}$. La Historia Natural, y, en concreto, la Botánica y la Química fueron consideradas ciencias útiles por los profesionales sanitarios ilustrados; la Botánica fue la ciencia útil por excelencia que repercutió significativamente en el proceso modernizador de las profesiones sanitarias del siglo XVIII: médicos, cirujanos y boticarios.

El proceso de modernización administrativa y docente de la sanidad culminó en los años finales del reinado de Carlos III. Concretamente, la Real Cédula de 13 de abril de 1780, que comenzó a regir en 14 de septiembre de 1780, estableció la separación de los asuntos profesionales de médicos, cirujanos y boticarios; dispuso que en el Real Tribunal del Protomedicato se dirigiesen y gobernasen por sí mismas las tres Facultades de Medicina, Cirugía y Farmacia, teniendo cada una sus Audiencias separadas, haciendo cada una de ellas sus exámenes, y que administrasen justicia en sus asuntos respectivos ${ }^{30}$. Según Folch Andreu ${ }^{31}$, fue el hecho de más categoría de la Historia de la Farmacia.

A raíz de tal disposición, el Real Jardín Botánico de Madrid se estableció como único centro docente donde los boticarios podían adquirir una formación científica en Botánica, y se proyectaba también para impartir clases de Química y Farmacia ${ }^{32}$. En lo concerniente a la Botánica, con anterioridad a 1780 hubo ya actividad docente; el Real Decreto de 17 de noviembre de 1762 estableció la asistencia obligatoria de los oficiales y mancebos de botica a las clases impartidas ${ }^{33}$. Establecida la división del Real Tribunal del Protomedicato de 1780, la formación de los boticarios continuó al margen de la universidad, pero fue más científica; la Audiencia de Farmacia recibió las competencias de la capacitación científica de los boticarios aprendices, quedando gobernada por el Protofarmacéutico, que era el Boticario Mayor de la Real

29 Moreno GonzÁlez, A. (1988), Progreso, secularización e instrucción pública, Revista de Occidente, 82, pp. 6-27.

30 IBORRA, P. (1987), Historia del Protomedicato en España (1477-1822) (edición, introducción e índices de Juan Riera y Juan Granda-Juesas). En Riera Palmero, J. (dir.), ActaHistorio-Médica Vallisoletana, XXIV, Universidad de Valladolid, Secretariado de Publicaciones, pp. 77-79.

31 Folch ANDreu (1935), Los farmacéuticos en la Academia durante el siglo XVIII. Labor de D. José Hortega. En Academia Nacional de Medicina 1734-1934. Publicaciones conmemorativas del II centenario de su fundación, Madrid, Imprenta de J. Cosano, pp. 177-282.

32 Calleja Folguera (1988), pp. 161-171; Puerto Sarmiento (1988), pp. 66-72; Puerto SARMiento (1989), La enseñanza de la Farmacia en la España Ilustrada, OFFARM, 8 (2), pp. 61-67.

33 Vidal Casero, M.C. (1981), Inicios de las lecciones de Botánica en el Jardín Botánico de Migas Calientes y Jardín Botánico del Prado. OFFARM, 2 (11), pp. 525-531. 
Botica, y tres Alcaldes examinadores, dos de ellos ayudas de la misma institución y uno de los Maestros del Real Jardín Botánico ${ }^{34}$.

Botánica y Farmacia confluyeron con el inicio de los cursos de Botánica en el Real Jardín Botánico de Madrid el 16 de mayo 178135. El Reglamento de 17 de marzo de 1783 estableció docencia botánica general y aplicada para médicos, cirujanos y boticarios ${ }^{36}$, reglamento que ha sido comentado por Puig Samper ${ }^{37}$. Casimiro Gómez Ortega (catedrático primero) y Antonio Palau Verdera (catedrático segundo) se alternaron en la docencia a partir de 1784. El curso general de Botánica impartido por el primero comenzó el primero de abril de este año, estando matriculados 153 alumnos, y concluyó el 31 de julio de $1784^{38}$. El día primero de septiembre del mismo año comenzaron las demostraciones prácticas, explicando este catedrático las plantas que tienen uso en la Medicina, y particularmente aquellas que no se habían explicado durante el curso de primavera y verano por no haber florecido ${ }^{39}$.

Volviendo a los párrafos de José García Ramos, y teniendo en cuenta los cinco párrafos anteriores, se puede precisar que Vicente Cervantes Mendo obtuvo el título de boticario en Madrid conforme a la modernización administrativa y docente de la sanidad derivada de la Real Cédula de 13 de abril de 1780, que comenzó a regir cuando tenía 22 años de edad. Es cierto que obtuvo el título de boticario en Madrid, pues así consta en su solicitud de Título de Colegial Honorario, que envió desde México al Real Colegio de Boticarios de Madrid con fecha de 2 de octubre de 1794, solicitud que se conserva en el Archivo de la Real Academia Nacional de Farmacia, reproducida en un trabajo anterior ${ }^{40}$; afirma en ella ser «Boticario revalidado por el Real Protomedicato de Madrid». Sin embargo, ni en su solicitud ni el informe de Casimiro Gómez Ortega de 21 de septiembre de 1786 hay fecha de aprobación de boti-

34 Calleja Folguera (1988), p. 163; Puerto Sarmiento (1988), p. 68; Francés CauSAPÉ, M.C. (1989), La farmacia durante el reinado de Carlos III, An. Real Acad. Farm., 55, pp. 74-92.

35 Puerto SARMiento (1988), p. 63.

36 Calleja Folguera (1988), pp. 175-177.

37 PUIG SAMPER (1987), La enseñanza de la Botánica en la España Ilustrada. En SÁNCHEZ, B, Puig-SAmPer, M.A. y Sota, J. De la (eds.), La Real Expedición Botánica a Nueva España, 1787-1803, Madrid, Comisión Quinto Centenario/Real Jardín Botánico/CSIC, pp. 59-78.

38 Memorial Literario, Instructivo y Curioso de la Corte de Madrid, Madrid, Imprenta Real, abril de 1784, pp. 28-38.

39 Memorial Literario, Instructivo y Curioso de la Corte de Madrid, Madrid, Imprenta Real, septiembre de 1784, pp. 8-9.

40 Pastor Villegas y Pastor Valle (2003), p. 105. 
cario; no fue antes de 1784 pues Bellot Rodríguez ${ }^{41}$ encontró una lista de los alumnos que fueron a examen a la Facultad de Farmacia del Real Protomedicato este año con certificación de Casimiro Gómez Ortega de haber aprobado la Botánica en el Real Jardín Botánico de Madrid, en la cual figura Vizente Zervantes (sic). Así pues, no hay duda de que su formación botánica fue en el nuevo emplazamiento de tal institución, realizando después el examen establecido.

He revisado si entre las instancias de mancebos de botica solicitando examen de Botánica en 1784 figura la de Vicente Cervantes Mendo alegando motivos de no asistencia a clase o de asistencia irregular. No figura tal instancia en el Archivo del Real Jardín Botánico de Madrid. En otras palabras, no constan las vicisitudes que José García Ramos menciona en el aprendizaje de la Botánica antes de comparecer a examen.

Vicente Cervantes Mendo cumplió los requisitos vigentes entonces para obtener el título de boticario, que están recogidos en estudios que se han ocupado de la formación de boticarios durante la Ilustración Española ${ }^{42}$. Resumidamente, el aspirante a boticario debía iniciar su formación siendo admitido por un boticario aprobado para trabajar como aprendiz en su botica o en la botica hospitalaria que regentase. El boticario aprendiz, cumpliendo los requisitos de edad (tener cumplidos veinticinco años), limpieza de sangre (ser hijo legítimo de padres honrados y cristianos viejos), conocimiento de latín y acreditar haber practicado cuatro años con maestro aprobado, comparecía a examen (teórico y práctico) ante el Tribunal de Farmacia del Real Protomedicato con acreditación de haber aprobado la Botánica en el Real Jardín Botánico de Madrid. Este tribunal revalidaba o no al boticario aprendiz para ejercer la profesión.

Casimiro Gómez Ortega destaca en su informe de 21 de septiembre de 1786 que Vicente Cervantes tuvo una formación inicial interdisciplinar. En mi opinión, además de la formación botánica en el Real Jardín Botánico de Madrid, resulta verosímil que consiguiera una formación farmacéutica clínica mucho más amplia que la formación teórica y práctica que se podía conseguir en cualquier botica pública.

41 Bellot Rodríguez, F. (1972), Algunos boticarios y mancebos de botica alumnos del Real Jardín Botánico del Prado en 1784, An. Real Acad. Farm., 38, pp. 611-623.

42 Calleja Folguera, M.C. (1988), p. 5; Puerto Sarmiento (1989), pp. 61-67; PeRAlTA REgLADO, J.M. (2005), Las obras para la instrucción de los boticarios en la España del siglo XVIII: Análisis y aportaciones, Madrid, pp. 16-24; Peralta-REGladO, J.M. y GoMIS, A. (2006), Las cartillas farmacéuticas publicadas en España en el siglo XVIII, Ars Pharm, 47, pp. 23-26. 
Los Reales Hospitales de la Corte, General y de la Pasión, estaban cercanos al Real Jardín Botánico de Madrid, formando parte de las instituciones científicas matritenses en torno al actual Paseo del Prado. El Real Hospital General de Madrid, inicialmente institución asistencial de hombres, aglutinó en sus dependencias tres instituciones docentes en el siglo XVIII: el propio Hospital, el Real Colegio de Cirugía de San Carlos (a partir del 1 de octubre de 1787) y el Estudio Real de Medicina (a partir de 1795) ${ }^{43}$.

Folch Andreu ${ }^{44}$ afirmó que la formación de boticarios en la Botica de los Reales Hospitales General y de la Pasión de Madrid fue una realidad en el siglo XVIII. El Archivo Regional de la Comunidad de Madrid conserva las Constituciones y Ordenanzas de 1760 de los mencionados hospitales, reimpresas en 178045; el capítulo IX corresponde a la Botica en sus veintisiete apartados, siendo competencia del Boticario Mayor la docencia (apartado 16). Dicho capítulo ha sido reproducido y comentado por Martínez Tejero et al. ${ }^{46}$, quienes consideran que no es aventurado afirmar que se estudiaron los efectos de los medicamentos en la misma cabecera del enfermo. Se ha apuntado también por Francés Causapé y Martínez Crespo $^{47}$ que la función del boticario responsable de la Botica del Real Hospital General de Madrid tuvo una mayor importancia clínica y asistencial gracias al asesoramiento del insigne boticario José Hortega y Hernández.

Tras la inauguración de la parte construida en 1781 (gran patio y edificio ocupado hoy por el Centro de Arte Reina Sofía) del magno proyecto de Fran-

43 Costa Carballo, C.M. Da (1992), La enseñanza de la Medicina a finales del siglo XVIII en las instituciones docentes madrileñas ubicadas en el Hospital General de Madrid, Madrid, Universidad Complutense de Madrid, p. 85; NúÑEZ OlARTE, J.M. (1999), El Hospital General de Madrid en el siglo XVIII (actividad médico-quirúrgica), Madrid, CSIC, pp. 19-64 y 287-308.

44 Folch ANDreu (1941), pp. 27-57.

45 Constituciones, Ordenanzas, para el Gobierno de los Reales Hospitales General, y de la Pasión de Madrid, aprobadas por el Rey nuestro señor Don Carlos Tercero (que Dios guarde) y pasadas por el Real Consejo de Castilla, Reimpresa en Madrid en la Imprenta de Pantaleón Aznar, Año de 1780.

46 Martínez Tejero, V., Mendaza Beltrán, M. y Peralta Sanz, M.T (1979), La farmacia clínica en los Reales Hospitales General y de la Pasión de Madrid, según las Constituciones y Ordenanzas aprobadas por Carlos III en 1760. En XXIII Congreso Nacional de la Asociación Española de Farmacéuticos de Hospitales: Zaragoza, octubre 1978, Zaragoza, Asociación Española de Farmacéuticos de Hospitales, pp. 259-264.

47 FrancÉs CAusapé, M.C y Martínez CRespo, C. (1999), El farmacéutico José Ortega y Hernández y las Constituciones y Ordenanzas del Hospital General y de la Pasión de Madrid, An. Real Acad. Farm., 65, pp. 643-666. 
cisco Sabatini, en el Hospital General de Madrid había 38 salas (24 para hombres y 14 para mujeres) y un total de 1561 camas $^{48}$. Tal vez, Vicente Cervantes Mendo fuese aprendiz de boticario en este hospital con anterioridad a 1784, pudiendo conocer también la actividad médico-quirúrgica del hospital, en donde fueron boticarios aprendices y luego boticarios distinguidos del mismo sus amigos Antonio de la Cruz Martín y Benito Pérez Valdés. Ambos son mencionados con afecto por Vicente Cervantes Mendo en la carta que envió al primero de ellos, fechada en México el 1 de abril de 1818, reproducida en un trabajo anterior ${ }^{49}$.

Concerniente a las obras destinadas a la instrucción de boticarios en el siglo XVIII, ninguna de ellas fue publicada por una institución oficial ${ }^{50}$; son breves en extensión y profundidad de contenidos, pareciendo más adecuadas para artesanos que para científicos ${ }^{51}$. Las dos primeras ediciones del Examen Pharmaceutico del boticario Francisco Brihuega, publicadas en Madrid en 1761 y 1775-76, respectivamente, sirvieron para la formación teórica de los boticarios aprendices en el reinado de Carlos III en gran parte de España. Ambas ediciones, y otra posterior de 1796 publicada también en Madrid, están escritas en forma de diálogo (preguntas y respuestas); han sido analizadas en trabajos anteriores ${ }^{52}$. Por otra parte, las obras publicadas en Madrid Palestra Pharmaceutica Chymico Galenica (varias ediciones) del boticario Félix Palacios y Bayá, y la Farmacopoeia Matritensis (ediciones de 1739 y 1762) eran las guías para los boticarios y médicos de España y del Imperio español ${ }^{53}$. Todas estas publicaciones y otras, así como el ambiente profesional en que practicó Vicente Cervantes Mendo, debieron influir significativamente en su formación inicial, resaltada por Casimiro Gómez Ortega en su informe de 21 de septiembre de 1786.

48 García Barrero, P. (1997), El hospital general de Madrid (Parte III) -de Campomanes y Floridablanca, a nuestros días-, Arbor, 64, pp. 93-127; LAFUENTE, A. (1998), Guía del Madrid Cientifico, Madrid, Doce Calles/Comunidad de Madrid/ CSIC, pp. 153-157.

49 Pastor Villegas y Pastor Valle (2003), p. 69.

50 Peralta Reglado (2005), pp. 444-446.

51 González Bueno, A. y Puerto Sarmiento, F.J. (1989), Ciencia y Farmacia durante la Ilustración. En Sellés M., Peset, J.L. y LAfuente, A. (compiladores), Carlos III y la ciencia de la Ilustración, Madrid, Alianza Editorial, pp. 247-261.

52 GonzÁlez Bueno y FrancÉs CAusApé, M.C. (1991), Divulgación científica en la Farmacia Española Ilustrada: el Examen Pharmaceutico..., Llull 14, pp. 495-505; PerALTA Reglado, J.M. (2005); Peralta-Reglado, J.M y Gomis, A. (2006), Las cartillas farmacéuticas publicadas en España en el siglo XVIII, Ars Pharm, 47, pp. 23-26.

53 Tate Lanning, J. (1997), El Real Protomedicato (Díaz Córdoba, M.A, y Soberanes Fernández, J.L., trads.), México D.F., Universidad Nacional Autónoma de México, pp. 332-378. 
Tras su formación inicial, Vicente Cervantes Mendo compareció a examen ante el Tribunal de Farmacia del Real Protomedicato. A partir de 1781, se conocen los miembros (Protofarmacéutico y Alcaldes Examinadores) que formaron tal tribunal. Concretamente, los miembros del tribunal de 1784, año en que pasó a examen a la Facultad de Farmacia con certificación de Casimiro Gómez Ortega de haber aprobado la Botánica, figuran en la Tabla 1, elaborada con la información obtenida de una publicación oficial de ese año ${ }^{54}$. Juan Díaz figura como Protofarmacéutico y Casimiro Gómez Ortega como Alcalde Examinador perpetuo.

TABLA 1. Tribunal de Farmacia del Real Protomedicato del año 1784

\begin{tabular}{|l|l|l|}
\hline MIEMBRO DEL TRIBUNAL & CARGO EN EL TRIBUNAL & PROFESIÓN Y DOMICILIO \\
\hline Juan Díaz & Protofarmacéutico & $\begin{array}{l}\text { Boticario de S.M., calle y } \\
\text { casa del Tesoro }\end{array}$ \\
\hline Matías García Ros & Alcalde Examinador perpetuo & $\begin{array}{l}\text { Boticario de S.M., calle y } \\
\text { casa del Tesoro }\end{array}$ \\
\hline José Enciso & Alcalde Examinador perpetuo & $\begin{array}{l}\text { Boticario de S.M., calle } \\
\text { de Leganitos }\end{array}$ \\
\hline Casimiro Gómez Ortega & Alcalde Examinador perpetuo & $\begin{array}{l}\text { Primer Catedrático de } \\
\text { Botánica, calle de la } \\
\text { Montera }\end{array}$ \\
\hline Joaquín Antón y Jiménez & Alcalde Examinador perpetuo & $\begin{array}{l}\text { Asesor, calle de Barrio } \\
\text { nuevo }\end{array}$ \\
\hline José Pérez Caballero & Alcalde Examinador perpetuo & $\begin{array}{l}\text { Consejo de Hacienda, } \\
\text { Fiscal, calle del Arenal }\end{array}$ \\
\hline
\end{tabular}

4. Vinculación Profesional con el Real Hospital General de MADRID

Según José García Ramos, Vicente Cervantes consiguió por oposición ser nombrado boticario del Real Hospital General de Madrid tras actuar en los

54 Kalendario manual y Guía de forasteros en la Corte de Madrid para el año de M.DCC.LXXXIIII... [Madrid] (1784), Madrid, Imp. Real de la Gazeta, pp. 86-89. 
Ejercicios Públicos de Botánica celebrados en el Real Jardín Botánico de Madrid, lo cual es erróneo. Sus palabras sobre tal oposición fueron ${ }^{55}$ :

\begin{abstract}
Quiso la mala estrella de Cervantes que a poco tiempo le atacase una dolencia pertinaz que le imposibilitó dedicarse a trabajo alguno, viéndose obligado a retirarse a Alcalá de Henares, en cuya población creía encontrar y halló en efecto algún alivio a sus padecimientos. Pero aún estaba en bastante mal estado, cuando recibió un día carta de su maestro y amigo G. Ortega [Casimiro Gómez Ortega], en la cual le participaba que había ocurrido la vacante de la plaza de farmacéutico del Hospital de esta Corte, y que debiendo proveerse por oposición, era necesario que remitiese a la mayor brevedad posible la solicitud debida. [...]. Fue por consiguiente nombrado farmacéutico del Hospital General de Madrid.
\end{abstract}

Como se puede observar al leer este párrafo, no se precisa la vacante que se había producido y cuándo se cubrió por oposición. Referente a la Botica, estaba ordenado en las Constituciones, Ordenanzas, para el Gobierno de los Reales Hospitales General, y de la Pasión de Madrid, mencionadas en el apartado anterior, que la Junta de Gobierno de los Reales Hospitales de Madrid nombraría un Boticario Mayor aprobado por el Real Protomedicato; un Mancebo Mayor, también aprobado por dicho tribunal; un Mancebo del Almacén, un segundo Mancebo de Botica, bien instruido en la Facultad; y otros Mancebos.

La oposición que menciona José García Ramos no se debe confundir con los ejercicios públicos realizados anualmente por los mancebos Practicantes de Botica de los Reales Hospitales de Madrid aspirando a los premios ofrecidos por su Real Junta de Gobierno. En tales ejercicios se trataron temas de Química y Farmacia, pero no de Botánica ${ }^{56}$. En los primeros ejercicios, que se celebraron los días 14, 15 y 16 de abril de 1784, actuaron todos los mancebos Practicantes, estando entre ellos el ya mencionado Benito Pérez Valdés, quien actuó también en el año siguiente. Los censores de los ejercicios de los años 1784 y 1785 fueron Leoncio Álvarez y Francisco Icedo, Boticarios Mayores de tales hospitales. No se celebraron los ejercicios de 1786, por ser año de mucha enfermería, y continuaron en los años siguientes. Hay constancia de la actuación de Antonio de la Cruz Martín en los ejercicios celebrados en 1787, que correspondían al año anterior; los censores de esta convocatoria fueron Casimiro Gómez Ortega (Catedrático primero de Botánica del Real Jardín Botánico y Boticario Mayor Honorario de S.M.) y Benito Pérez Valdés

55 GARCÍA RAMOS (1864), pp. 8-9.

56 FolCh ANDREU (1941), pp. 27-57. 
(Mancebo Mayor), por enfermedad de Francisco Icedo. En los ejercicios celebrados en 1788, actuó también Antonio de la Cruz Martín, siendo ya boticario; los censores, además de Casimiro Gómez Ortega, fueron José Poblet (Director del Real Colegio de Boticarios) y los mencionados Francisco Icedo y Benito Pérez Valdés. Así pues, hay constancia de la actuación de los amigos de Vicente Cervantes, pero no de éste.

En el informe de Casimiro Gómez Ortega de 21 de septiembre de 1786 consta que Vicente Cervantes era boticario en esta fecha, y que en concurso público en el Real Hospital General de Madrid había merecido la preferencia sobre todos sus competidores. Así pues, la oposición a vacante fue anterior a la fecha de tan mencionado informe. Por consiguiente, rectificando lo escrito por José García Ramos, la oposición es anterior a los Ejercicios Públicos de Botánica celebrados en el Real Jardín Botánico de Madrid en diciembre de 1786, de los cuales se trata en la sección siguiente, y no tras actuar en ellos. Sin duda, Vicente Cervantes Mendo debió contar al opositar con el apoyo de Casimiro Gómez Ortega, quien estaba muy bien relacionado con los cercanos Reales Hospitales, General y de la Pasión.

Hay una razón significativa que apoya tal vinculación profesional. Morales Cosme y Aceves Pastrana ${ }^{57}$ han analizado las actividades de Vicente Cervantes en la Botica del Hospital General de San Andrés de México entre 1791 y 1808; concluyen que hizo rentable esta botica, y que ejerció la docencia aportando conocimientos de Farmacia, Botánica y Química para superar el modelo gremial de aprendizaje de los boticarios e iniciar el reconocimiento institucional de la profesión farmacéutica. En mi opinión, las observaciones que hizo en 1789 sobre la necesidad de adoptar un formulario, instrucción y adecuado empleo de los oficiales, así como de la disposición de los tres almacenes en el interior de la botica de dicho hospital, no las hubiera podido formular sin un conocimiento directo de la institución hospitalaria madrileña tenido en años anteriores.

\section{BOTÁNICO POR EL REAL JARDÍN BOTÁNICO DE MADRID}

Un aspecto importante del Reglamento del Real Jardín Botánico de Madrid de 1783 es la institucionalización de la figura del botánico ${ }^{58}$. A partir de

57 Morales Cosme, A. y Aceves Pastrana, P. (2003), Negocio, reglamentación y profesionalización farmacéutica: la botica del Hospital General de San Andrés (1770-1809), Montalbán, 36, pp. 45-64.

58 Puig SAMPer (1987), pp. 59-78; PUerto SARMiento (1988), pp. 71-72. 
esta fecha, botánico sería quien realizase los cursos, o bien quien la institución reconociese como tal, nombrándolo comisionado. El Real Tribunal del Protomedicato expediría el título de aprobación con la adición de Botánico a quienes, cumpliendo los requisitos para ser admitidos a examen en su Facultad correspondiente, presentasen certificación de haber asistido y aprovechado las clases; con tal adición tendrían preferencia, en igualdad de las demás circunstancias, en las vacantes de plazas propias de la profesión sanitaria en la Casa Real, Ejércitos y Hospitales Generales y de Guerra. La docencia, ajustada a los conocimientos linneanos, estaba dirigida fundamentalmente a los boticarios, pero entre los matriculados había muchos médicos, cirujanos, militares y eruditos, ya que el título de botánico tenía mérito preferente en las comisiones reales o del Real Tribunal del Protomedicato.

La Gaceta de Madrid anunció en 1783 la bibliografía botánica recomendada $^{59}$. Hasta diciembre de 1786, los textos publicados fueron: Principios de Botánica... de Miguel Barnades (Madrid, 1767), Disertación acerca de los métodos botánicos escrita en francés por el célebre Mr. Duhamel de Monceau y traducida e ilustrada... de Casimiro Gómez Ortega (Madrid, 1772), Tabulae Botanicae... de Casimiro Gómez Ortega (Madrid, 1773), Explicación de la filosofia y fundamentos botánicos de Linneo... de Antonio Palau (Madrid, 1778), Tablas botánicas en que se explican sumariamente las clases, secciones y géneros de plantas que trae Tournefort... de Casimiro Gómez Ortega (Madrid, 1783), y Curso Elemental de Botánica, teórico y práctico, dispuesto para la enseñanza del Real Jardín Botánico de Madrid de Casimiro Gómez Ortega y Antonio Palau Verdera (Madrid 1785).

José García Ramos ${ }^{60}$ menciona la distinción que Casimiro Gómez Ortega hizo a Vicente Cervantes al poco tiempo de ser examinado de boticario:

Desde aquel día, ya no fue Cervantes, para G. Ortega [Casimiro Gómez Ortega] el desconocido aspirante a boticario [...]. Deseoso de llamar hacia Cervantes la atención del público ilustrado y amante de las ciencias naturales, y dar al mismo tiempo una pública prueba de la justicia con que apreciaba su verdadero mérito, declinó G. Ortega sobre él la señalada honra de componer y pronunciar el discurso de apertura de las clases de Botánica en uno de los años siguientes. Llenó en esta ocasión Cervantes su cometido tan satisfactoriamente, que el Excmo. Sr. Duque de Osuna, comisionado por la Majestad del Rey Carlos III para presidir el acto en su Real nombre, informó a éste del triunfo obtenido por Cervantes, en tales términos

59 Gaceta de Madrid, Madrid, 20 de mayo de 1783, n. 40; Gaceta de Madrid, Madrid, 3 de junio de 1783, n. 44.

60 GARCÍA RAMOS (1864), p. 8. 
que creyó justo el mismo tomar la determinación de mandar que el discurso se imprimiese y publicase a sus expensas. Y no pareciéndole esta medida suficiente recompensa para nuestro compañero, ni suficiente estímulo para sus ulteriores progresos en la ciencia, le hizo la señalada merced de regalarle un ejemplar de su propio discurso, lujosamente encuadernado, en el cual había puesto de su Real puño una dedicatoria al autor.

Los días 6 y 9 de diciembre de 1786 se celebraron los Ejercicios Públicos de Botánica dirigidos por Casimiro Gómez Ortega. No se celebraron el 6 de julio de ese año, como figura en publicaciones de José Luis Maldonado Polo (tal vez por error involuntario) que tratan de José Longinos Martínez Garri$\mathrm{do}^{61}$. La Figura 1 muestra la portada del impreso de tales ejercicios ${ }^{62}$, portada que ha sido publicada en trabajos previos ${ }^{63}$, en la que figuran los alumnos elegidos para actuar; las demás páginas detallan sus profesiones, los asuntos señalados a cada uno de ellos y los alumnos elegidos para dificultar.

El primer párrafo del mencionado impreso explicita la intención de tales ejercicios (prueba pública del adelantamiento de los cursantes de Botánica), el criterio de selección de los alumnos cursantes de Botánica para actuar y dificultar (asistencia con regularidad a las clases y haberse distinguido en ellas por su aprovechamiento), y el texto seguido en las clases (Curso elemental de Botánica, de Casimiro Gómez Ortega y Antonio Palau Verdera, publicado en 1785).

Las profesiones de los tres primeros actuantes constan en las páginas interiores del impreso: José Longinos era cirujano, y Gregorio Bacas y Vicente Cervantes eran boticarios. La profesión de Andrés Cuellar no consta, pero en el impreso de los ejercicios públicos de Botánica celebrados en 1788 consta que era presbítero, y aprobado en Botánica y Farmacia ${ }^{64}$. Los alumnos elegidos para dificultar a los cuatro actuantes son los que figuran en la Tabla 2, elaborada con los datos del impreso y la información del acto proporcionada por la Gaceta de Madrid65.

61 Maldonado Polo (1997), p. 136; Maldonado Polo (1997), De California a El Petén. El naturalista riojano José Longinos Martínez en Nueva España, Logroño, Ediciones Instituto de Estudios Riojanos, p. 24; MALDONADO POLO (2001), p. 107.

62 Exercicios Públicos de Botánica (1786), Madrid, Imprenta Real.

63 Puig Samper (1987), p. 73; Pastor Villegas y Pastor Valle (2003), p. 64.

64 Memorial Literario, Instructivo y Curioso de la Corte de Madrid, Madrid, Imprenta Real, septiembre de 1788, pp. 69-76.

65 Gaceta de Madrid, Madrid, 19 de diciembre de 1786, n.101. 


\title{
EXERCICIOS PÚBLICOS DE BOTANICA,
}

\author{
QUE TENDRÁN
}

EN LA PIEZA DE LA ENSENANZA

DE LAS CASAS

\section{DEL REAL JARDIN BOTÁNICO}

DON JOSEPH LONGINOS, DON GREGORTO BACAS, DON VICENTE CERVANTES, $X$ DON ANDRES CUELI.AR;

\section{DIRIGIENDOLOS}

EL D. D. CASIMIRO GOMEZ ORTEGA, primer Catedratico de Botanica con hanores de Boticario Mayor de S. M. , y Alcalde Examinador perpetuo de Pliarmacia en el Real Protomedicato.

FL MIERCOLES 6 Y SABADO 9 DE DICIEMBRE

A LAS 3 DE SUS TARDES,

RN MADRID EN LA IMPRENTA REAL.

$$
1786 \text {. }
$$

Figura. 1. Portada del impreso de los Ejercicios Públicos de Botánica celebrados en el Real Jardín Botánico de Madrid en el año 1786. 
TABLA 2. Alumnos del Real Jardín Botánico de Madrid que dificultaron en los Ejercicios Públicos de Botánica de 1786.

\begin{tabular}{|l|l|}
\hline ALUMNO DIFICULTADOR & PROFESIÓN \\
\hline Antonio Pineda & Oficial del Real Cuerpo de Guardias Españolas \\
\hline Jaime Miralles & Doctor en Leyes \\
\hline José Albarrán & Bachiller en Medicina \\
\hline José Regato & Cirujano aprobado \\
\hline Pedro Pérez del Carmen & Religioso de la Orden de San Juan de Dios \\
\hline Julián Gutiérrez & Cirujano aprobado \\
\hline Benito Pérez Valdés & Boticario aprobado \\
\hline Jorge del Castillo & Boticario aprobado \\
\hline Blas Esteban Gómez & Practicante de Farmacia \\
\hline
\end{tabular}

De acuerdo con el impreso de los ejercicios de 1786, a Vicente Cervantes Mendo se le señalaba para tratar en su actuación de los asuntos que figuran en el párrafo que sigue:

D. Vicente Cervantes explicará en particular los caracteres de la Clase séptima y siguientes hasta la duodécima inclusive, y los de sus respectivos Órdenes: determinará y describirá la especie o especies de ellas que se le indiquen: expondrá las leyes de la vegetación, o sea la germinación de la semilla, el crecimiento de la planta, su fecundación, los varios estados por donde pasa hasta la maduración del fruto, y los diversos medios naturales o artificiales de multiplicar y propagar los vegetales. Defenderá asimismo con qué contribuyen hasta cierto grado muy apreciable las luces de la Botánica combinada con las de la Física y de la Chímica (sic) a la indagación de las propiedades de las plantas; es, a saber, así de las generales como de las económicas y medicinales. Glosará los aforismos en que trata de Linneo de los nombres, de las diferencias, de las variedades y de las virtudes. Por último, responderá a las dudas y objeciones que se han publicado en varios tiempos contra el sistema del docto Naturalista Carlos Linneo, que se han adoptado en esta Escuela y Jardín, y en general contra el modo de estudiar sistemáticamente la Botánica.

A los pocos días de celebrados estos ejercicios, las publicaciones periódicas de Madrid Mercurio Histórico y Político ${ }^{66}$ y Memorial Literario y Curioso de la Corte de Madrid ${ }^{67}$ dieron también informaciones sobre los mismos. La segunda de ellas recoge que los ejercicios no se celebraron en el mes de julio,

66 Mercurio Histórico y Político, Madrid, diciembre de 1786, pp. 357-358.

67 Memorial Literario, Instructivo y Curioso de la Corte de Madrid, Madrid, Imprenta Real, diciembre de 1786, pp. 360-362. 
es decir, al finalizar el curso de primavera-verano, pues fueron aplazados hasta el regreso de la Corte a Madrid. Se celebraron solemnemente, asistiendo ambos días el Excmo. Sr. Protector del Real Jardín Botánico, conde de Floridablanca, y numerosas personalidades nacionales y extranjeras. También recoge el discurso pronunciado por Vicente Cervantes Mendo el primer día, antes de iniciarse las actuaciones de los cuatro alumnos seleccionados, en el que mencionó el momento de la Botánica en España, América Española y Filipinas, dejó entrever la Real Expedición Científica a la Nueva España (1787-1803), y mencionó también la próxima inauguración del Real Laboratorio de Química como complemento del Real Jardín Botánico de Madrid y destacó los Reales Hospitales de Madrid:

A vista de tantos, y tan eficaces auxilios dispensados por el Rey nuestro Señor a la Botánica, de tan loables, y distinguidos ejemplos suministrados por nuestros Españoles; de la benignidad con que V.E. se digna repetirnos el honor de presenciar como Protector de esta escuela, los ejercicios anuales de sus discípulos, y de los premios y honrosas distinciones que les ha conseguido de la Soberana piedad: ¿quién habrá ya que dude de que el Jardín Botánico de Madrid colocado como felizmente se halla dentro del recinto de la población, a corta distancia de su centro, en la vecindad de un Palacio Real, y del más frecuentado y magnífico paseo, y en la inmediación de los Reales Hospitales Generales, donde por el celo, y acertadas disposiciones de su Real Junta se educa tan numerosa, y floreciente juventud, competirá dentro de poco, sino se aventaja en hermosura, número, y variedad de plantas, y copia de frutos de la enseñanza, con los más famosos de Europa? Pero V.E. va de una vez a añadir a su obra el complemento: la Academia de las Ciencias con su laboratorio Químico, y observatorio astronómico, que inmortalizará el Reinado de Carlos el Sabio [Carlos III] trasladando a la posteridad [...], y estimada de sus hermanas las demás naciones del Universo.

Es de señalar que pocos meses antes de su disertación se informaba de la publicación de la Oración apologética por la España y su mérito literario... ${ }^{68}$, conocida y estudiada obra cuyo autor es el hombre de letras extremeño Juan Pablo Forner. Sin embargo, fue Vicente Cervantes Mendo quien precisa claramente el momento científico-político español y sus perspectivas de futuro en base al Real Jardín Botánico de Madrid y a su entorno científico de 1786.

A Vicente Cervantes Mendo, primero de los actuantes, le preguntaron y mandaron describir las plantas que eligieron Antonio Pineda, Jaime Miralles, José Albarrán, José Regato y Pedro del Carmen ${ }^{69}$. Estos cursantes dificultaron

68 Memorial Literario, Instructivo y Curioso de la Corte de Madrid, Madrid, Imprenta Real, octubre de 1786, pp. 360-362.

69 Memorial Literario, Instructivo y Curioso de la Corte de Madrid, diciembre de 1786, p. 500. 
también a José Longinos Martínez Garrido ${ }^{70}$, quien fue el segundo de los actuantes. Es relevante que el primero de estos alumnos es Antonio de Pineda Ramírez, quien, además de ser militar de profesión (marino), fue el naturalista en la Expedición Malaspina (1789-1794); estuvo encargado de la Historia Natural hasta su muerte en Filipinas en $1792^{71}$.

Así pues, precisando lo escrito por José García Ramos, Vicente Cervantes Mendo actuó como primer alumno distinguido en los segundos ejercicios públicos de Botánica que se celebraron con gran solemnidad los días 6 y 9 de diciembre de 1786 en el Real Jardín Botánico de Madrid. Ambos días asistió el Excmo. Sr. Protector del Real Jardín Botánico, José Moñino y Rodríguez, conde de Floridablanca, presidiendo el acto en nombre de Carlos III, y no el Excmo. Sr. Duque de Osuna. Casimiro Gómez Ortega y todos los discípulos que intervinieron como actuantes y dificultadores cumplimentaron el día 10 de diciembre de 1786 al conde de Floridablanca.

Se puede observar en la Figura 2 que los cuatro actuantes solicitaron conjuntamente el título de aprobación en Botánica tras la celebración de los ejer$\operatorname{cicios}^{72}$. Es relevante que Vicente Cervantes Mendo y José Longinos Martínez Garrido pasaron, junto con sus esposas, al año siguiente a Nueva España $^{73}$. Es también relevante que Vicente Cervantes Mendo y Antonio de Pineda Ramírez, condiscípulos del Real Jardín Botánico de Madrid, coincidieron en el Real Jardín Botánico de México transcurridos cinco años; Vicente Cervantes Mendo le entregó un manuscrito que había redactado referente a la flora medicinal, calificado de «discurso elocuente» por Antonio de Pineda Ramírez ${ }^{74}$ y éste escribió al maestro común Casimiro Gómez Ortega el 19 de diciembre de 1791 desde la corbeta Descubierta, surta en el puerto de Acapulco y dispuesta para zarpar hacia las Islas Marianas, congratulándose de condiscípulo tan aventajado tanto por sus conocimientos como por su trato y conducta $^{75}$.

70 Maldonado Polo (1997), p. 24; Maldonado Polo (1997), p. 136; Maldonado POLO (2001), p. 107.

71 Beddall, B. (1983), Antonio Pineda. En López PiÑero, J.M., Glick, T.F., NAVArro Brotóns, V. y Portela Ramos, M., Diccionario histórico de la ciencia moderna en España, Barcelona, Ediciones Península, vol. 2, pp. 177-178.

72 Archivo del Real Jardín Botánico de Madrid, I, 13, 6, 7.

73 Pastor Villegas; Pastor Valle (2003), p. 65.

74 GonZÁlez Claverán, V. (1989), Aportación novohispana a la Expedición Malaspina. En Peset, J.L. (coord.), Ciencia, Vida y Espacio en Iberoamérica, Madrid, CSIC, vol. I, pp. 427-437.

75 Archivo del Real Jardín Botánico de Madrid, I, 20, 4, 29. 
Dos años después, Benito Pérez Valdés fue uno de los actuantes y Antonio Cruz Martín asistió para argüir ${ }^{76}$. Así pues, ambos boticarios fueron también alumnos distinguidos del Real Jardín Botánico de Madrid. No asistió el compañero y amigo Vicente Cervantes Mendo, porque ya se encontraba en México dedicado a la expedición botánica novohispana.

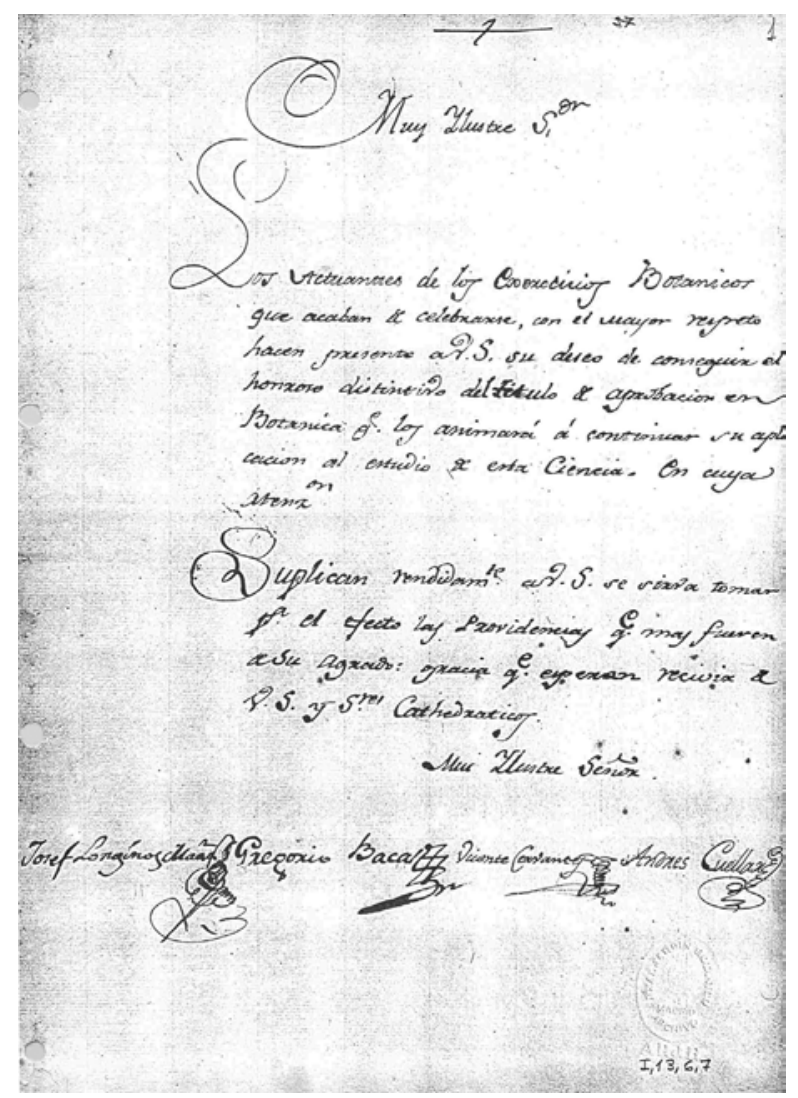

Figura 2. Solicitud conjunta del título de botánico al Real Tribunal del Protomedicato firmada por José Longinos Martínez, Gregorio Bacas, Vicente Cervantes y Andrés Cuellar.

76 Memorial Literario, Instructivo y Curioso de la Corte de Madrid, Madrid, septiembre de 1788, pp. 69-76. 


\section{CONCLUSIONES}

De todo lo expuesto anteriormente, se deducen las cuatro conclusiones siguientes sobre la formación científica y ejercicio profesional en España del insigne científico hispanomexicano Vicente Cervantes Mendo:

1. Se formó profesionalmente como boticario y botánico en el Madrid de los años finales del reinado de Carlos III, llegando a tener vinculación profesional con el Real Hospital General de Madrid.

2. Pasó a examen a la Facultad de Farmacia del Real Tribunal del Protomedicato con certificación de Casimiro Gómez Ortega, catedrático primero del Real Jardín Botánico de Madrid, de haber aprobado la Botánica en 1784, consiguiendo la aprobación de boticario. En el tribunal examinador de este año figuran Juan Díaz como Protofarmacéutico y Casimiro Gómez Ortega como Alcalde Examinador perpetuo.

3. Se puede concretar que tuvo vinculación profesional con el Real Hospital General de Madrid con anterioridad al 21 de septiembre de 1786, antes de obtener el título de botánico. Sin duda, en esta institución hospitalaria consiguió una formación farmacéutica clínica relevante, y conoció la actividad médico-quirúrgica propia del hospital.

4. Como alumno distinguido del Real Jardín Botánico de Madrid, pronunció un discurso y actuó en primer lugar en los Ejercicios Públicos de Botánica realizados solemnemente los días 6 y 9 de diciembre de 1786, obteniendo el título de botánico. En el discurso pronunciado repasó el momento político-científico español. En su actuación explicó lo que se le señaló en el impreso de tales ejercicios, en particular la defensa de la combinatoria científica Botánica-Física-Química a la indagación de las propiedades de las plantas (generales, económicas y medicinales).

Fecha de recepción: 23 de agosto de 2008

Fecha de aceptación: 5 de febrero de 2009 